\title{
A Case of Hair Regrowth in a Patient with Complete Androgen Insensitivity Syndrome and Female Pattern Hair Loss
}

\author{
Luigi Laino* \\ MD Dermatologist and Venereologist, Director of Dermatologic Centre of Rome "Latuapelle” Via Bixio, Rome, Italy
}

*Corresponding author: Laino L, MD Dermatologist and Venereologist, Director of Dermatologic Centre of Rome "Latuapelle" Via Bixio, 95 001185, Rome, Italy, Tel/Fax: +390645550661; E-mail: info@latuapelle.it

Received: 18 Dec, 2019 | Accepted: 02 Jan, 2020 | Published: 09 Jan, 2020

Citation: Laino L (2020) A Case of Hair Regrowth in a Patient with Complete Androgen Insensitivity Syndrome and Female Pattern Hair Loss. J Clin Cosmet Dermatol 4(1): dx.doi.org/10.16966/2576-2826.145

Copyright: (C) 2020 Laino L. This is an open-access article distributed under the terms of the Creative Commons Attribution License, which permits unrestricted use, distribution, and reproduction in any medium, provided the original author and source are credited.

\section{Key points}

There are new scientific evidences that suggest thinking about a new etiological terminology for FPHL not linked to hyperandrogenism and secondary signs of masculinization. This study could create the interpretative model that confirms how the Female Pattern Hair Loss (Ludwig clinical classification), unrelated to hyperandrogenism and secondary signs of masculinization, could be linked eminently to a hormone-receptor alteration of the estrogenic type.

\begin{abstract}
Androgen Insensitivity Syndrome (AIS) is a rare hormonal condition associated with disorders of sexual development resulting in a variable phenotype. The AIS is classified in Partial Androgen Insensitivity (PAIS) and Complete Insensitivity to Androgens (CAIS). Hair loss in the female model, also known as Female Androgenetic Alopecia (FPHL), has usually been considered an androgen-dependent disorder that represents the female counterpart of male pattern baldness. However, most women with FPHL have no other signs or symptoms of hyperandrogenism and have normal androgen levels, indicating that our understanding of the pathogenesis of the disorder remains incomplete. We describe a female phenotypic-type hair loss that occurs in a patient with Complete Androgen Insensitivity Syndrome (CAIS) characterized by a 5-alpha-reductase 2 steroid deficiency, in which we performed a non-hormonal combined restorative hair therapy. (Low Laser/Led Light+local sub-dermal infiltration of hair growth factor) and topical minoxidil 5\%, which led to an excellent result in hair regrowth after 6 months. In light of these new data, an alternative potential androgen-independent mechanism for FPHL phenotype deserves to be carefully considered, as well as rethinking the identifying term of this type of baldness, in estrogenetic alopecia.
\end{abstract}

Keywords: Alopecia; Dermatoscopy; Genetics; Hair loss; Trichoscopy; Female pattern hair loss

\section{Introduction}

Androgen Insensitivity Syndrome (AIS) is a rare hormonal condition associated with a sexual developmental disorders resulting in varying phenotypes [1]. These disorders of androgen action present as $46 \mathrm{XY}$ Disorders or Differences of Sex Development (DSD). The phenotypic spectrum of AIS depends on the residual androgen receptor activity and encompasses individuals with completely female phenotype to male phenotype with infertility /undervirilization. AIS is classified into Partial Insensitivity to Androgens (PAIS), and complete insensitivity to androgens (CAIS) where coexists in CAIS a deficiency of 5 alpha reductase type 2, which codes for Dihydrotestosterone (DHT), whose secondary deficit, is held responsible for phenotypic feminization.

Female pattern hair loss, also known as female androgenetic alopecia, is a non-scarring progressive thinning of hair [2]. It results from a progressive decrease in the ratio of terminal hairs to shorter, thinner vellus hairs, a process known as follicular miniaturization.
Although the etiology is controversial, FPHL has usually been considered as an androgen-dependent disorder representing the female counterpart of male balding. Further, some authors use the terms "androgen-dependent FPHL" and "androgen-independent FPHL" to separate women who have FPHL due to androgen excess from those with normal androgen levels [3]. However, most women with FPHL have no other signs or symptoms of hyperandrogenism and have normal androgen levels, indicating that our understanding of the pathogenesis of the disorder remains incomplete [4].

It is known that the switching of testosterone to dihydrotestosterone encoded by the enzyme 5 alpha reductase has always been considered the main way to lead to miniaturization of hair, both in male pattern baldness and in FPHL [5].

The differentiation of CAIS and PAIS from 5-alpha-reductase is by analyzing the ratio of testosterone to DHT in serum. In patients with steroid 5-alpha-reductase 2 deficiency, the production of DHT decreases, and the plasma ratio of testosterone to DHT increases. 
In most girls/boys with PAIS, the production of DHT, and the ratio of testosterone to DHT is normal. Women with CAIS, may have secondary 5-alpha-reductase deficiency due to a decreased mass of the urogenital tract tissues, which normally produces DHT [6].

We describe a phenotypic female pattern hair loss occurred in a patient with Complete Androgen Insensitivity syndrome (CAIS) characterized by a steroid 5-alpha-reductase 2 deficiency, in which we have performed a combined non-hormonal hair restoration treatment (Low Laser/Led Light+sub-dermal local infiltration of hair growth factor) [7], followed by daily application of topical minoxidil 5\% solution, which resulted in an excellent result in hair regrowth after 6 months.

\section{Case Report}

A Medical Doctor Colleague 28-year-old girl, carrier of Complete Androgen Insensitivity Syndrome (CAIS), due to absence of uterus and menstruation, comes to our attention, presenting a 10-year history of gradual thinning of her scalp hair.

On clinical examination, she presents a diffuse reduction in hair density especially in the front-temporal region, with diffuse thinning of the crown region, with preservation of the frontal hairline. The scalp skin appears slightly atrophic, with the presence of some benign keratotic lesions.

At Videodermoscopy, widespread miniaturization of hair appeared within the analyzed area, associated with centro-follicular depression and reduction of local density, as well as a significant alteration of the average hair diameter.

The diagnosis of phenotypic FPHL (Model Ludwig, grade II) was therefore carried out.

The patient was therefore subjected to routine hormonal tests which showed a non detectable level of DHT $(<0.001 \mathrm{nM} / \mathrm{L})$.

Following genetic and molecular examinations, the patient demonstrated the classic 46XY phenotype associated to a Complete Insensibility to Androgens (CAIS) in addition to a deficiency of 5 alpha reductase type 2 .

\section{Materials and Methods}

In this study, androgenetic alopecia was diagnosed clinically and based on the Ludwig scales and VideoTrichoscopy (DermaView ${ }^{\circ}$ ) patterns [8].

The Patient gave her written informed consent to publish this case (including publication of images).

The patient received on their bald areas (Figure 1), a Combined Low Laser Light Phototherapy and Growth Factor Hair Formulation Infiltration Therapy (AGA Combo Treatment) [7] consisting of a micro-infiltration session every 3 weeks with non-pharmacological/ hormonal therapy containing a Restructuring Hair Booster-natural Growth Factor Formulation (GFF) industrial product with:

Uncross-linked hyaluronic acid

Fibroblast growth factors+polypeptide+NaDNA (N-acetyl-2,3dehydro-2-deoxyneuraminic acid)

Amino acids: arginine, alanine, aspartic acid, glutamic acid, glycine, histidine, hydroxyproline, isoleucine, lysine, methionine, phenylalanine, proline, serine, threonine, tyrosine, leucine, valine;

Trace elements: $\mathrm{Si}, \mathrm{Ca}, \mathrm{Fe}, \mathrm{K}, \mathrm{Mg}, \mathrm{Mn}, \mathrm{Na}, \mathrm{P}, \mathrm{SE}$;

Vitamins: B12, B9, B3, B5, A, E, B8-Terpenes: ginkgolides A,B,C,M, Bilobalide;

Fatty acids: Linoleic acid, Oleic acid

Flavonoids: Quercetin, Kaempferol

Antioxidants: Vitamin E, Chlorogenic acid, Gallic acid, Quercetin, Kaempferol

The micro-infiltration sessions were immediately followed by a 30-minute sessions (2 sessions per month for 24 weeks) of LLLT therapy (LLLT device:-Kernel $\left.{ }^{\circledR} \mathrm{KN}-7000 \mathrm{~A}\right)$ using LED Photodynamic Therapy-Technical data during a single treatment:-Session Time: 20 minutes-Range of emission: $633 \mathrm{~nm} \pm 10 \mathrm{~nm}$-Power of emission: $80 \mathrm{~mW} / \mathrm{cm}^{2} \pm 20 \%$-Type of emission: continue emission.

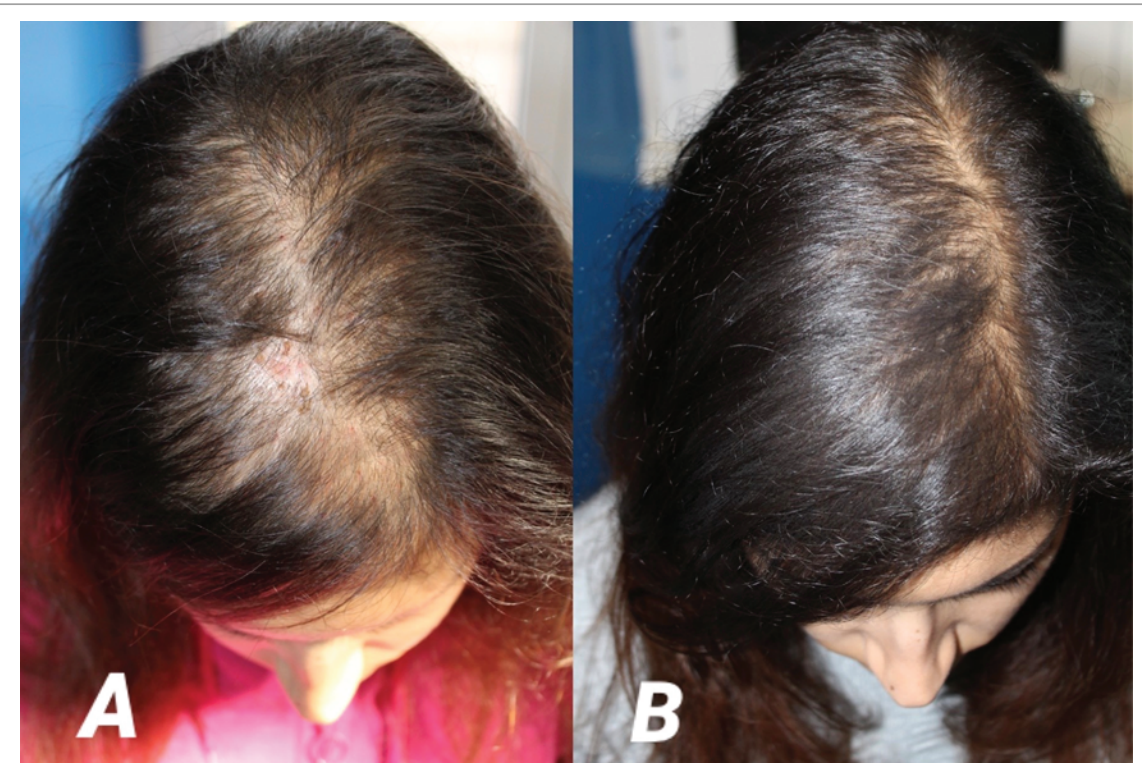

Figure 1: Clinical images before (A) and after (B) treatment. Notice the excellent hair restoration, in terms of thickening of the fronto-parietal area after 6 months of therapy. .. 
The patient underwent home therapy with industrial topical minoxidil 5\% solution, once a day on the front-parietal scalp area.

Due to the peculiar nature of this study it is not required to provide any statement of the institutional review committee or the approval of the committee of human subjects.

\section{Results}

The results obtained after 6 months, revealed a clinical (Figure 1), and trichoscopic (Figure 2), significantly higher percentage of recovery from androgenetic alopecia.

The patient shows an excellent increase of diameter and density of hair, with reduction of perifollicular atrophy; no side effects in terms of pain, itching, tingling, discomfort, edema, excoriations, necrosis, fissures, erosions, eczema, were detected in the patient, both in the very next days and in the short to medium term.

\section{Discussion}

Androgen insensitivity syndrome arises from loss-of-function mutations in the coding sequence of the Androgen Receptors (AR). This X-linked genetic mutation of the androgen receptor gene results in the dysfunction of androgen receptors and hormone resistance. These mutations lead to a loss in virilization or infertility in 46XY males in individuals with functional testes and adequate testosterone production. CAIS and PAIS encompass variability in phenotypic expression; however, both these conditions have similar genetic, endocrine, and pathophysiologic mechanisms.

Androgen insensitivity syndrome is the result of profound resistance of the androgen receptor towards the action of androgen. Various studies done in women established the Androgen Receptor (AR) dysfunction with no detectable androgen receptor binding leading to resistance to the virilization effect of the exogenous androgen. The androgen receptor nuclear receptor contains a hormone-binding domain and an N-terminal region used for transactivation, and most mutations cause androgen receptor dysfunction to localize specifically in the hormone-binding domain [1].
Our case described a CAIS Syndrome: There are various forms of presentation of CAIS in different age group, varying from infants to adolescent females. CAIS will present very commonly as primary amenorrhea in adolescent females or incidental finding of testes in females undergoing inguinal hernia repair. Physical examination reveals a female phenotype at birth, which will be a mismatch from the results of prenatal fetal sexing (Y chromosome on DNA analysis). The vagina is blind-ending with no uterus, gonads present in the lower abdomen or inguinal canal. At puberty, with the normal growth spurt, there is breast development secondary to the conversion of androgens to estrogens, and taller female phenotype secondary to the effect of the $\mathrm{Y}$ chromosome.

17-beta-hydroxysteroid dehydrogenase deficiency: CAIS and PAIS individuals have normal testosterone responses after the hCG stimulation test. Low serum testosterone synthesis implies impaired testosterone synthesis secondary to 17-beta-hydroxysteroid dehydrogenase deficiency

5-alpha-reductase deficiency: differentiation of CAIS and PAIS from 5 -alpha-reductase is by analyzing the ratio of testosterone to DHT in serum. In patients with steroid 5-alpha-reductase 2 deficiency, the production of DHT decreases, and the plasma ratio of testosterone to DHT increases. In most girls/boys with PAIS, the production of DHT, and the ratio of testosterone to DHT is normal. Women with CAIS may have secondary 5-alpha-reductase deficiency due to a decreased mass of the urogenital tract tissues, which normally produces DHT [6].

In serum, Testosterone $(\mathrm{T})$ is bound to albumin and in part to SHBG (Sex-Hormone Binding Globulin) and only a small fraction (2\%) circulates in free form, biologically active and therefore responsible for the effect at the level of target organs, following the specific link with the Androgen Receptor (AR). T can be converted by means of $5 a$-reductase to DeHydroTestosterone (DHT), which is the main mediator of androgenic effects in some target organs, including the prostate, seminal vesicles and external genitalia. The $\mathrm{T}$ can also be converted by Estradiol Aromatase (E2), which activates the specific

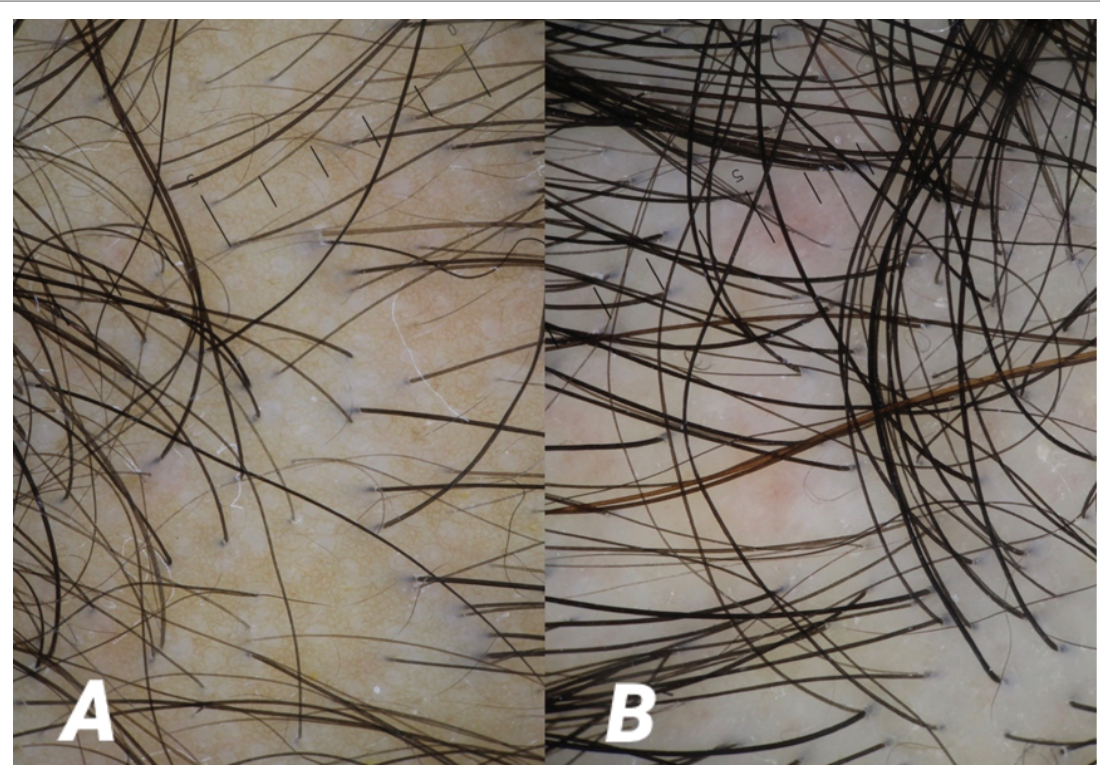

Figure 2: Trichoscopic images before $(A)$ and after treatment. Notice the marked reduction of miniaturization within the analyzed area, and the local hair densification, as well as a significant normalization of medium hair diameter. 
Estrogenic Receptors (ER $\alpha$ and $E R \beta$ ), suggesting the possibility that the biological action of $\mathrm{T}$ can take place not only through the activation of $\mathrm{AR}$, but also for ER activation. It is also known that ER $\beta$ receptors are present at the level of the pilosebaceous follicle; it is shown that the interaction of $\mathrm{T}$ with ER $\beta$ receptors at this level inhibits the growth and replication of hair follicle cells [9].

Although it has been assumed that androgenetic alopecia is the result of the abnormal sensitivity of hair follicles of the scalp to circulating androgens, in light of these new data, an alternative potential androgen-independent mechanism for FPHL phenotype deserves to be carefully considered.

As explained by Cousen P, et al. [10] and Langan EA, et al. [11] there are alternative hypothesis could explain a FPHL in CAIS.

Firstly it is possible that sufficient androgen levels, can directly operate to generate a phenotypic FPHL, but the lack of any secondary signs of virilization in patients with CAIS tends to exclude this hypothesis.

Secondly, testosterone (notoriously not active as a miniaturizing hormone lacking its active form, DHT) may be aromatized into estrogen, by the Cytochrome P-450 on the pilosebaceous follicle, which notoriously expresses the ER $\beta$ receptor for estrogens, which in turn, are known to inhibit the growth of hair in animal models, confirming the hypothesis of a miniaturizing action on the hair follicle, mediated by estrogen hormones.

Third, the non-pharmacological regenerative combined treatment (Low Level Laser Therapy [12]+sub-dermal micro-infiltration of non hormonal hair growth factors complex [7] that has been used for 6 months, does not act in a hormonal way, but only stimulating the reproduction of hair follicle germinative cells, through various described routes.

Minoxidil [13], is a potassium channel opener, causing hyperpolarization of cell membranes and it is also a vasodilator, it is speculated that, by widening blood vessels and opening potassium channels, it allows more oxygen, blood and nutrients to the follicle. Minoxidil also appears to stimulate the Vessel Endothelial Growth Factor (VEGF), which could promote the production of mesenchymal cells and anagen phase, at the level of the sub-follicular capillary microcirculation. However, despite the mechanism by which Minoxidil promotes hair growth is not fully understood, it is confirmed that minoxidil does not act as a mimetic-hormonal or promoting androgenic hormonal actions.

\section{Limitations}

The limits of this study are related to the low number of cases of CAIS with FPHL so far analyzed; the probable existence of CAIS not linked to FPHL could hypothesize that in some subjects there is a genotypic variability related to the hyper-expressivity of ER $\beta$ receptor expressed in hair follicle, or other pathogenetic pathways related to the onset of FPHL.

\section{Conclusion}

This case of a phenotypic FPHL highlights four incontrovertible conditions, such as:

The complete insensibility to androgens

The deficiency of 5 alpha reductase type 2

The lack of DHT

The excellent response in term of regrowth of hair after a non androgenic therapy
Taken together, these points support and confirm the hypothesis that the mechanism that led to FPHL could be an alternative to the androgen pathway, which has always been identified as the main one, even in female pattern baldness not clearly associated with secondary traits of hyper-androgenic manifestation (such as hypertrichosis and hirsutism, most often linked to a male pattern baldness of pattern, with bi-temporal retraction of the gulfs); in fact FPHL has so far been seen as a female variant of the male androgenic form, rather than a separate entity, also from the hormonal point of view. Due to the wide open debate on the complete codification of the FPHL etiology, which may not follow an exclusive androgen pathway, this work could serve as a model for further studies.

Although this case does not prove that all the FPHL are certainly caused by an alternative route to the androgenic pathway, the incontrovertible evidence of a case of complete insensitivity to hormones genetically associated to an of 5 alpha reductase type 2 deficit, with an excellent response to a non-pharmacological/hormonal regenerative treatment, explains the accreditation of a new hypothesis that sees FPHL, rather as an estrogenetic alopecia, instead of the always considered hypothesis of female pattern of male androgenetic alopecia. Therefore, the term "Estrogenic Alopecia" (EA), could be identified in an exclusive way, all those FPHL not clearly associated with hyperandrogenism and/ or signs of masculinization in women.

If further studies confirmed this hypothesis, this case, could represent the milestone for certifying that the pathogenesis of Female Pattern Hair Loss, may not be limited of an androgenic nature, strengthening the concept already amply demonstrated that estrogen can actually inhibit hair shaft generation due to their interaction with ER $\beta$ receptor expressed in hair follicle $[14,15]$.

\section{Statement of Ethics}

The author declares that this work has been carried out and prepared under the protection of all ethical and deontological principles.

\section{Disclosure Statement}

The author grants the possibility of disclosure of this original article and its attachments and contributions.

\section{Funding Sources}

None.

\section{Institutional Review Board Approval}

Obtained.

\section{Author Contributions}

The author states that the entire work was carried out with his own contribution.

\section{Declaration of Helsinki}

The Author state that the research was conducted ethically in accordance with the World Medical Association Declaration of Helsinki.

\section{Conflict of Interest}

Author's Conflict of interest declaration: None conflict of interest.

\section{References}

1. Hughes IA, Davies JD, Bunch TI, Pasterski V, Mastroyannopoulou $\mathrm{K}$, et al. (2012) Androgen insensitivity syndrome. The Lancet 380 : 1419-1428. 
2. Olsen EA (2001) Female pattern hair loss. J Am Acad Dermatol 45: S70-S80.

3. Wilson JD, Griffin JE, Leshin M, MacDonald PC, JB Stanbury JB, et al. (1983) The androgen resistance syndromes: $5 \alpha$-reductase 2 deficiency, testicular feminization, and related disorders. New York: McGraw-Hill.

4. Vujovic A, Del Marmol V (2014) The female pattern hair loss: Review of etiopathogenesis and diagnosis. Biomed Res Int 767628.

5. Karrer-Voegeli S, Rey F, Reymond MJ, Meuwly JY, Gaillard RC, et al. (2009) Androgen dependence of hirsutism, acne, and alopecia in women: retrospective analysis of 228 patients investigated for hyperandrogenism. Medicine (Baltimore) 88: 32-45.

6. Singh S, llyayeva S (2019) Androgen Insensitivity Syndrome. StatPearls [Internet].

7. Laino L (2019) Combined Low Laser Light Phototherapy and Growth Factor Hair Formulation Infiltration Therapy in Androgenetic Alopecia (AGA Combo Treatment). J Clin Cosmet Dermatol 3.

8. Rudnicka L, Olszewska M, Waśkiel A, Rakowska A (2018) Trichoscopy in Hair Shaft Disorders. Dermatol Clin 36: 421-430.

9. Pelletier G, Ren L (2004) Localization of sex steroid receptors in human skin. Histol Histopathol 19: 629-636.
10. Cousen P, Messenger A (2010) Female pattern hair loss in complete androgen insensitivity syndrome. $\mathrm{Br} J$ Dermatol 162: 1135-1137.

11. Langan EA, Paus R (2010) Female pattern hair loss: beyond an androgenic aetiology? Br J Dermatol 163: 1140-1141.

12. Jimenez JJ, Wikramanayake TC, Bergfeld W, Hordinsky $M$, Hickman JG, et al. (2014) Efficacy and safety of a low-level laser device in the treatment of male and female pattern hair loss: A multicenter, randomized, sham device-controlled, double-blind Study. Am J Clin Dermatol 15: 115-127.

13. Goren A, Shapiro J, Roberts J, McCoy J, Desai N, et al. (2015) Clinical utility and validity of minoxidil response testing in androgenetic alopecia. Dermatol Ther 28: 13-16.

14. Yip L, Zaloumis S, Irwin D, Severi G, Hopper J, et al. (2012) Association analysis of oestrogen receptor beta gene (ESR2) polymorphisms with female pattern hair loss. $\mathrm{Br} J$ Dermatol 166: 1131-1134.

15. Redler S, Birch P, Drichel D, Hofmann P, Dobson K, et al. (2014) The oestrogen receptor 2 (ESR2) gene in female-pattern hair loss: replication of association with rs10137185 in German patients. Br J Dermatol 170: 982-985. 